\title{
Dust cloud lightning in extraterrestrial atmospheres
}

\author{
Christiane Helling ${ }^{1}$, Moira Jardine ${ }^{1}$, Declan Diver ${ }^{2}$, Sören Witte ${ }^{3}$ \\ ${ }^{1}$ SUPA, School of Physics and Astronomy, University of St Andrews, St Andrews, KY16 \\ 9SS, UK, \\ ${ }^{2}$ School of Physics and Astronomy, University of Glasgow, Glasgow G12 8QQ, UK \\ ${ }^{3}$ Hamburger Sternwarte, Gojenbergsweg 112, 21029 Hamburg, Germany
}

\begin{abstract}
Lightning is present in all solar system planets which form clouds in their atmospheres. Cloud formation outside our solar system is possible in objects with much higher temperatures than on Earth or on Jupiter: Brown dwarfs and giant extrasolar gas planets form clouds made of mixed materials and a large spectrum of grain sizes. These clouds are globally neutral obeying dustgas charge equilibrium which is, on short timescales, inconsistent with the observation of stochastic ionization events of the solar system planets. We argue that a significant volume of the clouds in brown dwarfs and extrasolar planets is susceptible to local discharge events and that the upper cloud layers are most suitable for powerful lightning-like discharge events. We discuss various sources of atmospheric ionisation, including thermal ionisation and a first estimate of ionisation by cosmic rays, and argue that we should expect thunderstorms also in the atmospheres of brown dwarfs and giant gas planets which contain mineral clouds.
\end{abstract}

Keywords:

\section{Introduction}

Until recently, clouds were believed to be unique to Earth-like planets and to the gas giant planets that are rather far away from their host star (like Jupiter, Saturn, Uranus in our solar system). Extrasolar planets are now a matter of fact and their diversity has increased over the last couple of years due to various ground based observational efforts like SuperWASP, HAT, TrES and regarding giant gas planets, and by the CoRot and the Kepler space mission with respect to Earth-like, low-mass planets. Compared to the 
solar system, however, many giant gas planets are orbiting their host star at very short distance. Observations have revealed that hazes appear in the upper atmospheres of such close-in planets, because the haze absorbing the stellar radiation during transit makes the planet appear larger than expected. The transit spectroscopy of HD 189733b presented in Pont et al. (2008) and in Sing et al. (2011) provides the first proof that small mineral particles do not only populate the highest layers of the terrestrial atmospheres but are also present in extrasolar Jupiters. Such direct observations of atmospheric dust have not yet been possible for brown dwarfs. Brown dwarfs have the same size and effective temperature as the gas-giants, and they have been subject to extensive direct spectroscopic observations as they are much more close by and, hence, it is easier to take direct spectroscopic measurements for brown dwarfs than for the majority of the exoplanets. High- and lowresolution spectra, reaching from the optical into the near-IR, were detected and compared to synthetic spectra of model atmosphere simulations (e.g. Stephens et al. 2009; Witte et al. 2011, Patience et al. 2012). Researchers are keen to reproduce both observed spectra and also each others model results, leading to dedicated bench mark efforts for example for dust cloud models (e.g. Helling et al. 2008). More often, we learn something new only if model simulations do not fit observations. Jones \& Tsuji (1997) compared their static model atmosphere results to late M-dwarf spectra. The synthetic spectra only started to be comparable to observations when the authors reduced individual element abundances artificially, arguing these element would be locked in dust grains and, hence, be not available to the formation of molecules. Saumon et al. (2006) showed that the Spitzer observation of ammonia $\left(\mathrm{NH}_{3}\right)$ indicates vertical mixing of hotter material into detectable layers, hence, a local chemical dis-equilibrium. Their chemical equilibrium model did not fit the observations unless they artificially reduced the $\mathrm{NH}_{3}$ abundances, arguing that the reaction timescale of $\mathrm{N}_{2}$ to $\mathrm{NH}_{3}$ is much slower than the convective mixing timescale.

Clearly, clouds play an important role in every atmosphere where they are forming because they consume elements, and by this, change the local gas-phase chemistry. Cloud particles have large radiation absorption cross sections and they therefore increase the greenhouse effects, hence affecting the local temperature. Furthermore, these cloud form at a highly convective environment which drives a vivid turbulence field that can initiate dust formation (Helling et al. 2001), and which increases relative velocities between grains. Clouds have been observed to produce discharge events like lightning 
and sprites in planet of our solar system that carry clouds. Therefore, we have good reasons to expect that cloud-forming extrasolar planets and brown dwarfs show similar electrostatic activities.

We summarise our model of mineral cloud formation (Sect.2) and discuss in Sect. 4 if mineral clouds could produce lightning-like discharge events. Section 3 describes collisional ionisation and ionisation by cosmic rays as sources of charge separation in mineral clouds.

\section{Mineral cloud formation in extrasolar planets and brown dwarfs}

Brown dwarfs and gas giant planets outside of our solar system are likely not to form cloud made only of liquid droplets, but their warmer atmospheres do allow solid dust particles to condense from the gas phase.

We have modelled the formation of such mineral clouds by describing seed formation (by homogeneous nucleation) followed by the growth of 13 silicate and oxide solids by 60 chemical surface reactions, evaporation, gravitational settling (rain out), convective element replenishment, and element conservation (Woitke \& Helling 2003, 2004; Helling \& Woitke 2006 Helling et al. 2008b). Our model calculations start from solar element abundances which are subsequently depleted by seed formation and the growth of the grain mantle. If the grains become thermally unstable and evaporation sets in, the element abundances will be enriched by those elements previously locked in grains. Both processes, element depletion and element enrichment, are non-uniform and individual for each involved element. Processes between dust particles that lead to a further increase in grain size, like for example coagulation, are not part of our kinetic dust model because coagulation acts at a much longer time scale. Coagulation is about $100 \times$ slower than the growth process by surface reactions, hence, the formation processes (nucleation and growth) will be much faster (Helling et al. 2008a).

The onset of dust formation is triggered by a nucleation process that strongly depends on the local gas temperature and a high supersaturation of the seed forming gas species which requires a temperature well below thermal stability. Typical supersaturation ratios of our nucleation species $\mathrm{TiO}_{2}$ are well above $10^{4}$ in the nucleation region of the cloud (Fig. 1 in Helling et al. $2008 \mathrm{~b})$. The onset of growth requires the growing material to be thermally stable only. The growth rate is determined by the inflow of the growing gas phase constituents, hence, it is proportional to the number density of the (grow-) contributing species and their velocity distributions. We refer 


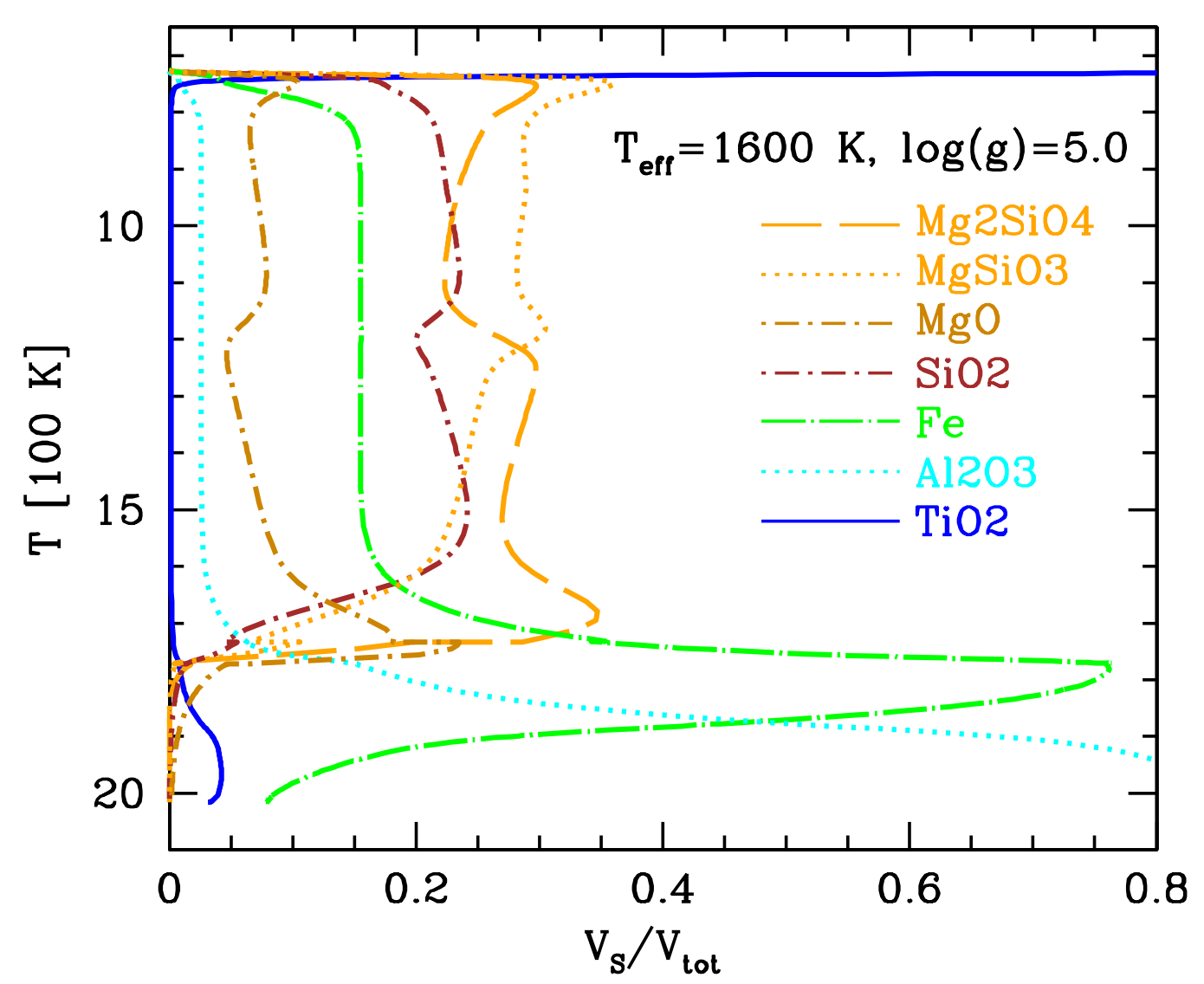

Figure 1: Dust cloud material composition in volume fractions $V_{\mathrm{s}} / V_{\text {tot }}$ in a giant gas planet atmosphere (Helling et al. 2008) as result of DRIFT-PhOENIX model atmosphere simulations that include our kinetic dust formation model (Witte et al. 2009; $\mathrm{T}_{\text {eff }}$ - effective temperature of object, $\log (\mathrm{g})$ - surface gravity of object). The composition changes with atmospheric height indicated by the local temperature.

to Helling \& Rietmeijer (2009) and above mentioned papers for more details regarding the model equations. Our solution of the kinetic dust formation predict a cloud structure as function of the local gas temperature and gas density, $T$ and $\rho_{\text {gas }}$. Our model predicts the mean grain size $\langle a\rangle\left(T, \rho_{\text {gas }}\right)$ $[\mu \mathrm{m}]$, the number density of dust particles $n_{\mathrm{d}}\left(T, \rho_{\text {gas }}\right)\left[\mathrm{cm}^{-3}\right]$, and the mean material composition of the cloud particles $V_{\mathrm{s}} / V_{\text {tot }}\left(T, \rho_{\text {gas }}\right)$ [\%] (e.g. Fig. 1). We also calculate the chemical composition of the gas phase, including the degree of ionisation (Sect. 4). $V_{\mathrm{s}}$ is the dust volume occupied by the solid species s, $V_{\text {tot }}$ is the total dust volume. To some extent, the mean grain size, 
number of dust particles, the total dust volume, and higher dust moments allows us to reproduce the grain size distribution, $f\left(V, T, \rho_{\text {gas }}\right)$ which provides the number of dust grains for each grain volume $V$.

Clouds in brown dwarfs and extrasolar giant gas planets are composed of a mixture of minerals due to the richness of the atmospheric precursor gas in these objects (Fig. 1), henceforth called 'mineral clouds'. The dust formation process (seed formation, growth/evaporation) is influenced by gravitational settling, hence, particle growth speeds up while the grains fall inward along a positive density gradient. During this descent, the crystal structure of the cloud particles is evolving (Helling \& Rietmeijer 2009). Figure 1 indicates that such clouds are made of small $\left(10^{-2} \mu \mathrm{m}\right)$ silicate particles at the top which develop into large $(10 \ldots 100 \mu \mathrm{m})$ iron $/ \mathrm{TiO}_{2}$ particles. For details on grain sizes see e.g. Fig. 8 in Helling et al. (2008b).

Would dust-dust collisions change this picture? The most interesting changes in the grain size distribution by a dust-dust collision likely result from the fragmentation of both collisional partners and the stick-and-hit events of projectile and target. Fragmentation would increase the number of grains and therewith the number of seeds for further grow. As surface growth is rather efficient, the grain fragments can grow rather quickly to their previous sizes until the gas-phase is undersaturated. Hence, dust-dust collisions tend to increase the number of grains but the grain size might not change as long as surface growth is efficient. Stick-and-hit events would produce a higher number of large grains, but the collision energetic need to be just right.

\section{Sources of mineral dust cloud ionisation}

The reasons for ionisation in clouds are rather diverse, and more complex processes than thermal ionisation need to be taken into account because of the rapidly decreasing gas temperature with height. They include energetic interstellar or interplanetary radiation, radioactive decay, differences between surface potentials of different materials (e.g. metals vs insulators), frictional ionisation, collisional ionisation of accelerated charges, or fragmentation of fractal particles (fracturing) . Triboelectric charging is suggested to be of particular interest for dust cloud charging on planetary surfaces (Sickafoose et al. 2001), a scenario which is rather similar to mineral clouds in substellar atmospheres. See also Saunders (2008) for an overview of the subject. Also, gas-phase ions can attach themselves to the grain surface, and by this, 
contribute to the charging of cloud particles. Nicoll \& Harrison (2010) have demonstrated this with observation of Earth clouds.

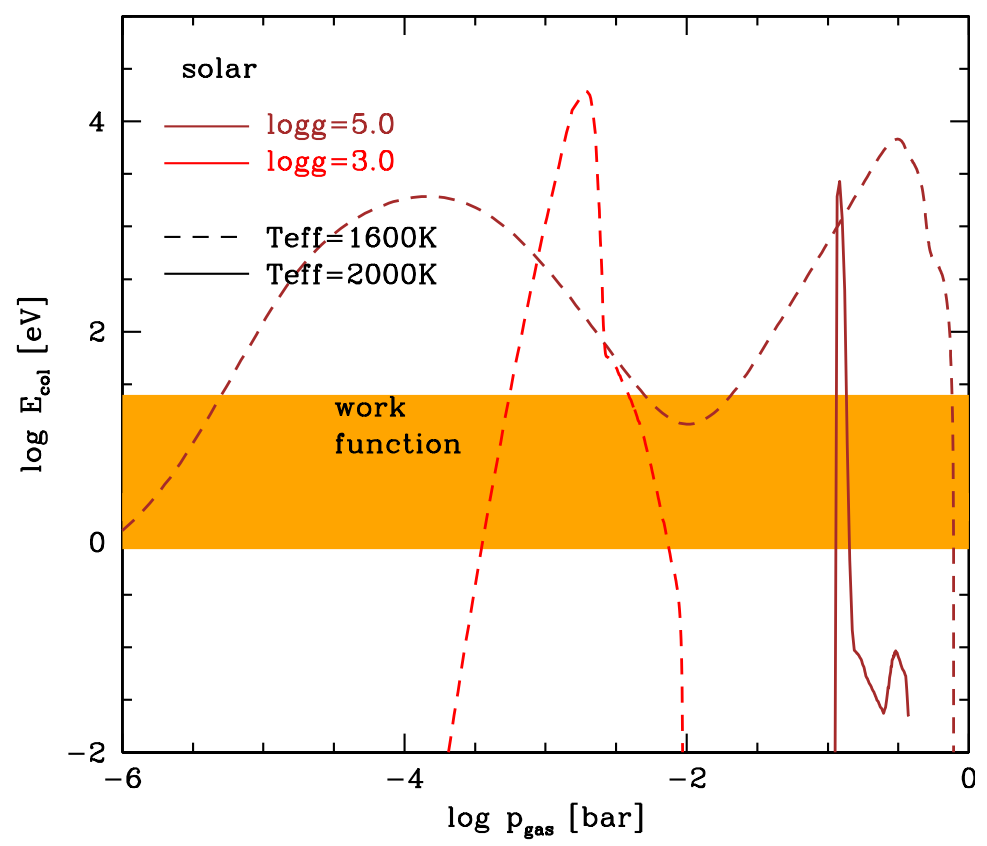

Figure 2: Turbulence enhanced dust-dust collision energies, $E_{\text {col }}$, for three different DRIFTPhoEnIX model atmosphere simulations $\left(p_{\text {gas }}\right)$. The collision energy is well above the work function interval (orange bar) for the high-gravity, cool brown dwarf $\left(\mathrm{T}_{\text {eff }}=1600, \mathrm{~K}\right.$, $\log (\mathrm{g})=5.0)$ over a large pressure range in contrast to its hotter counterpart $\left(\mathrm{T}_{\text {eff }}=2000\right.$, $\mathrm{K}, \log (\mathrm{g})=5.0)$.

In this paper, we discuss two sources of dust ionisation that both result in the ionisation of the cloud particles and eventually also in the ionisation of the gas phase.

\subsection{Dust-dust collisions}

Helling et al. (2011b) studied dust-dust collision kinematics using results of our kinetic dust formation model from the model atmospheres code Drift-Phoenix (Dehn 2007, Helling et al. 2008b, Witte et al. 2009). This collisional charge process is know as tribo-electric charging and is suggested to work well for dust charging of Martian dust (Sickafoose et al. 2001). Our results have shown that dust-gas and dust-dust collisions due to gravitational settling (drift, rain out) are not energetic enough to overcome the 

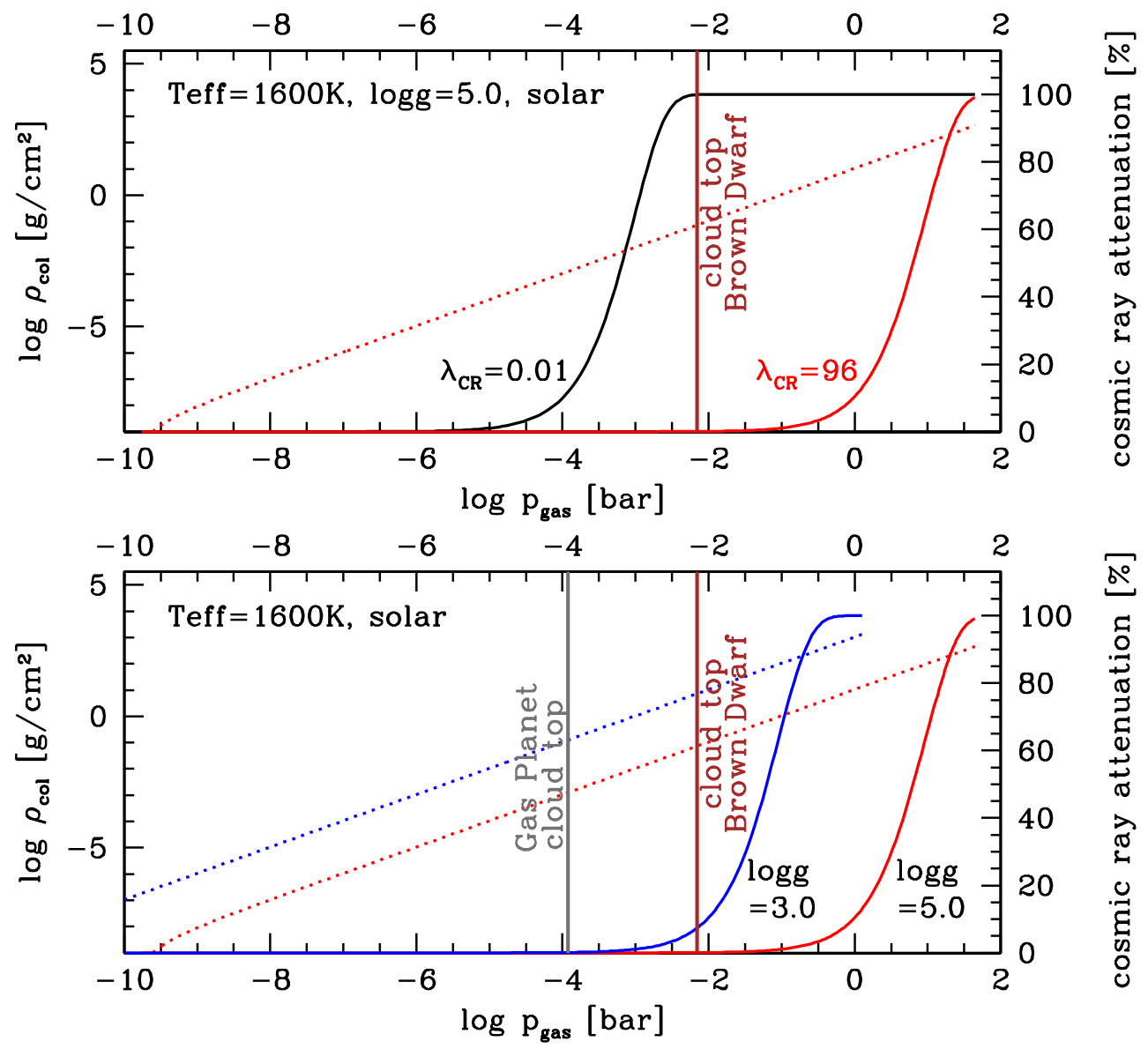

Figure 3: Cosmic ray attenuation (in \%; solid lines, right axis) and gas-phase column density $\rho_{\text {col }}$ (in $\mathrm{g} \mathrm{cm}^{-2}$; dotted lines, left axis). Top: The cosmic ray attenuation is shown for two cases in a brown dwarf DRIFT-PHOENIX atmosphere $\left(\mathrm{T}_{\text {eff }}=1600 \mathrm{~K}, \log (\mathrm{g})=5.0\right.$, solar metallicity) with $\mathrm{R}_{*}=10^{9} \mathrm{~cm}=0.14 \mathrm{R}_{\text {Jupiter }}$. $\lambda_{\mathrm{CR}}=96 \mathrm{~g} \mathrm{~cm}^{-2}$ (red solid line) where $\mathrm{H}_{2}$ provides the majority of the absorbing mass (Umebayashi \& Nakano 2009) and $\lambda_{\mathrm{CR}}=0.01 \mathrm{~g} \mathrm{~cm}^{-2}$ for which CR attenuation reaches $100 \%$ above the cloud top above which grains do form, too, but at a much lower rate. $\lambda_{\mathrm{CR}}$ is the characteristic CR penetration length. Bottom: Same as above but comparing a brown dwarf and a gas planet atmosphere. 
energy needed to release an electron from the crystal structure (work function). Only turbulence-enhances dust-dust collisions such that tribo-electric charging is possible.

Figure 2 shows the turbulence-enhanced energy by dust-dust collisions for three different atmosphere simulations. This rather limited number of DRIFT-PhOENIX investigated atmosphere models suggests that the pressure range affected by tribo-electric charges is larger in a compact, low temperature brown dwarf $\left(\mathrm{T}_{\text {eff }}=1600 \mathrm{~K}, \log (\mathrm{g})=5.0\right)$ compared to a hot brown dwarf $\left(\mathrm{T}_{\text {eff }}=2000 \mathrm{~K}, \log (\mathrm{g})=5.0\right)$ or a giant gas planet $\left(\mathrm{T}_{\text {eff }}=1600 \mathrm{~K}, \log (\mathrm{g})=3.0\right)$. The geometrical extension of the clouds, however, differ widely between brown dwarfs and giant gas planets. Brown dwarfs have much less extended clouds then giant gas planets due to their much higher surface gravity, and hence smaller pressure scale height. This suggests a smaller atmospheric volume being affected by charged mineral clouds in brown dwarfs compared to Jovian plants. However, our comparison of streamer timescale and Coulomb recombination time scale with the time scale on which dust particles pass through a previously formed electron cloud (streamer), and hence, potentially initiate another electron avalanche, suggest a stronger lightning activity in higher density environments such as brown dwarf atmospheres.

\subsection{Cosmic ray attenuation}

Cosmic rays (CR) appear to be an important source of atmospheric ionisation in the solar system planets. Observations of Earth clouds, however, suggest that the actual charge production is not overly efficient but potentially important for coagulation processes. Nicoll \& Harrison (2010) determine a maximum mean droplet charge of $17 \mathrm{e}$ at the cloud edges on Earth which can be directly related to ionisation by cosmic rays. The charging of these water cloud particles is, however, not a direct result of the impact of the high energy CRs on the cloud but rather of the ion and electron currents that develop from the $\mathrm{CR}$ ionisation of the gas above the cloud. These charges attach to the cloud particles. A similar scenario can be envisioned for close-in exoplanets that form an ionosphere due to the X-ray and the extreme UV radiation of the host star as demonstrated by Koskinen et al. (2010).

The question is whether galactic cosmic rays could be a global source of ionisation for the whole atmosphere of extrasolar low-mass objects which are not exposed to the high-energy radiation of a nearby host star. Ionisation by CRs includes the effect of secondary particles like electrons but also $\gamma$-rays. 
Umebayashi \& Nakano (2009) show for protoplanetary disks that the CR attenuation increases exponentially with a characteristic length or column density $\lambda_{\mathrm{CR}} \sim 96 \mathrm{~g} \mathrm{~cm}^{-2}$ of a gas where $\mathrm{H}_{2}$ provides the majority of the mass. This translates into a simple ansatz for the attenuation,

$$
\frac{F(z)}{F_{0}}=\exp \left(-\frac{\rho_{\mathrm{col}}}{\lambda_{\mathrm{CR}}}\right)
$$

with $F(z)$ the local CR flux at height $z, F_{0}$ the incident CR flux, and $\rho_{\text {col }}$ the gas column density. Figure 3 shows $F(z) / F_{0}$, the attenuation of cosmic rays (solid line, rhs. axis of figure), which is determined by the local atmospheric gas-phase column density $\rho_{\text {col }}=\int_{z_{0}}^{z_{\mathrm{i}}} \rho_{\text {gas }}(z) d z\left[\mathrm{~g} / \mathrm{cm}^{2}\right]$ (dotted line, lhs. axis of figure) integrated over the atmosphere extension $z_{0} \ldots z_{\mathrm{i}}$. The attenuation increases inward (downward) with increasing gas-phase column density.

Cosmic rays penetrate the cloud almost unhindered if $\mathrm{H}_{2}$ is the only absorber $\left(\lambda_{\mathrm{CR}}=96 \mathrm{~g} \mathrm{~cm}^{-2}\right.$ in Fig. 3). The gas column density is high enough for complete attenuation only at very high gas pressures in both the brown dwarf and the gas giant. Brown dwarfs, on the other hand, have been shown to maintain magnetic fields (e.g. Reiners \& Basri 2008; Hallinan et al. 2008; Christensen, Holzwarth \& Reiners 2009; Berger et al. 2010) and also planets have a magnetosphere (e.g. Zarka et al. 2001, Jardine \& Cameron 2008). Dolginov \& Stepinski (1993) argue that the magnetic field will diffuse the cosmic rays paths through the atmosphere and, hence, decrease the critical column density (or scale height) $\lambda_{\mathrm{CR}}$ for cosmic ray attenuation. Glauser et al. (2009) demonstrate that fast protons and He ions interact with dust grains in disks, hence, the dust will decrease the effective attenuation length in the atmosphere further. This suggests that a smaller fraction of the atmosphere and of the cloud is influenced by cosmic rays. Nontheless, the critical column density needs to be as low as $\lambda_{\mathrm{CR}}=0.01 \mathrm{~g} \mathrm{~cm}^{-2}$ (Fig. 3 , black solid line) to achieve $100 \%$ attenuation above the cloud in the brown dwarf model atmosphere studied here. It is, therefore, not clear if cosmic rays can be a global source for cloud ionisation as brown dwarfs have magnetic fields which are particularly strong on small scales. The B-field shielding would, however, be less efficient in the polar regions if the magnetic field is predominantly dipolar, an assumption which is justified for low-mass objects (see Morin et al. 2010). The consequence would be an increased polar discharge activity which is triggered by an increased degree of cloud ionisation due to CRs. 


\section{Should we expect thunderstorms in mineral dust clouds?}

Brown dwarf atmospheres are the perfect example for a neutral atmosphere if thermal ionisation is considered only (Mohanty et al. 2002). Figure 4 shows the degree of thermal ionisation of the gas phase in the pressure interval where the cloud forms for three different atmosphere models. The degree of ionisation increases inward towards higher temperature due to thermal ionisation but drops rather quickly in the low pressure regime. This figure demonstrates that the degree of thermal ionisation, $f_{\mathrm{e}, \text { thermal }}=p_{\mathrm{e}, \text { thermal }} / p_{\text {gas }}$, is less than $10^{-8}$. It is important to understand that although thermal ionisation does not provide enough free charges to produce, for instance, a magnetic Reynolds number $>1$, which would indicate the atmospheres potential for coupling to the strong magnetic field of brown dwarfs, the number of free electron may be large enough to produce a streamer between two charged dust grains or a whole front of charged dust grains (Dowds et al. 2003, Helling et al. 2011a,b)

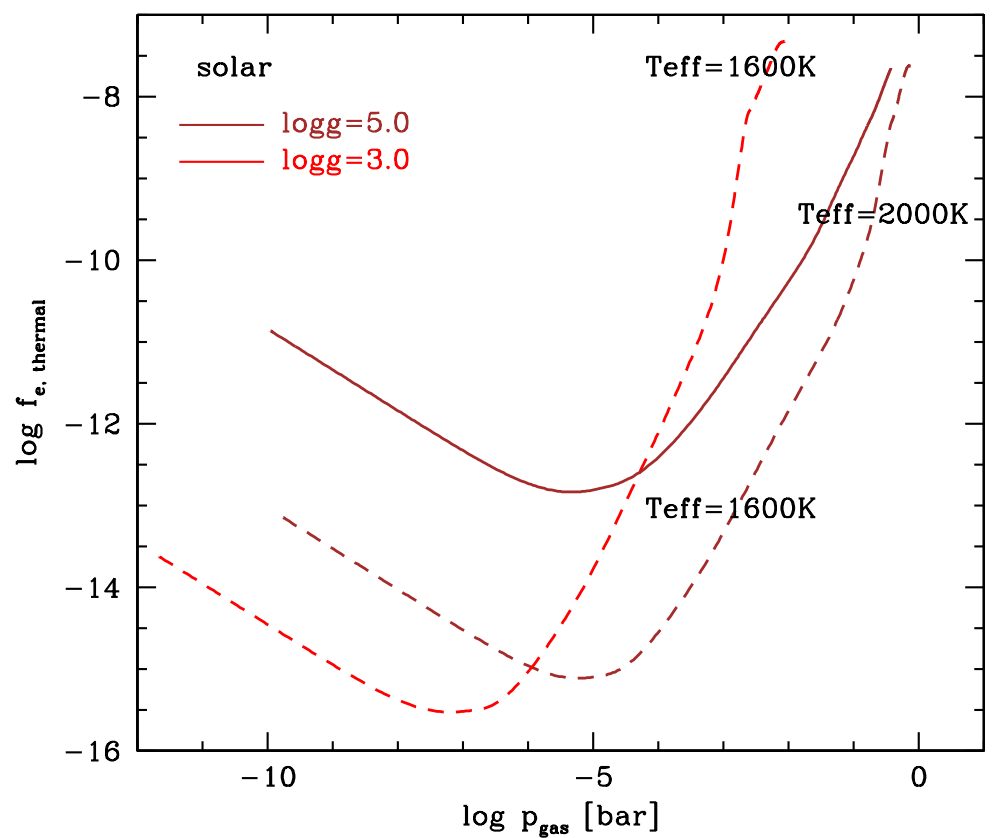

Figure 4: Degree of thermal gas-ionisation, $f_{\mathrm{e}, \text { thermal }}=p_{\mathrm{e}, \text { thermal }} / p_{\text {gas }}$, in the cloud for the same three example model atmospheres like in Fig. 2. ( $\mathrm{T}_{\text {eff }}$ - effective temperature of object, $\log (\mathrm{g})$ - surface gravity of object). 


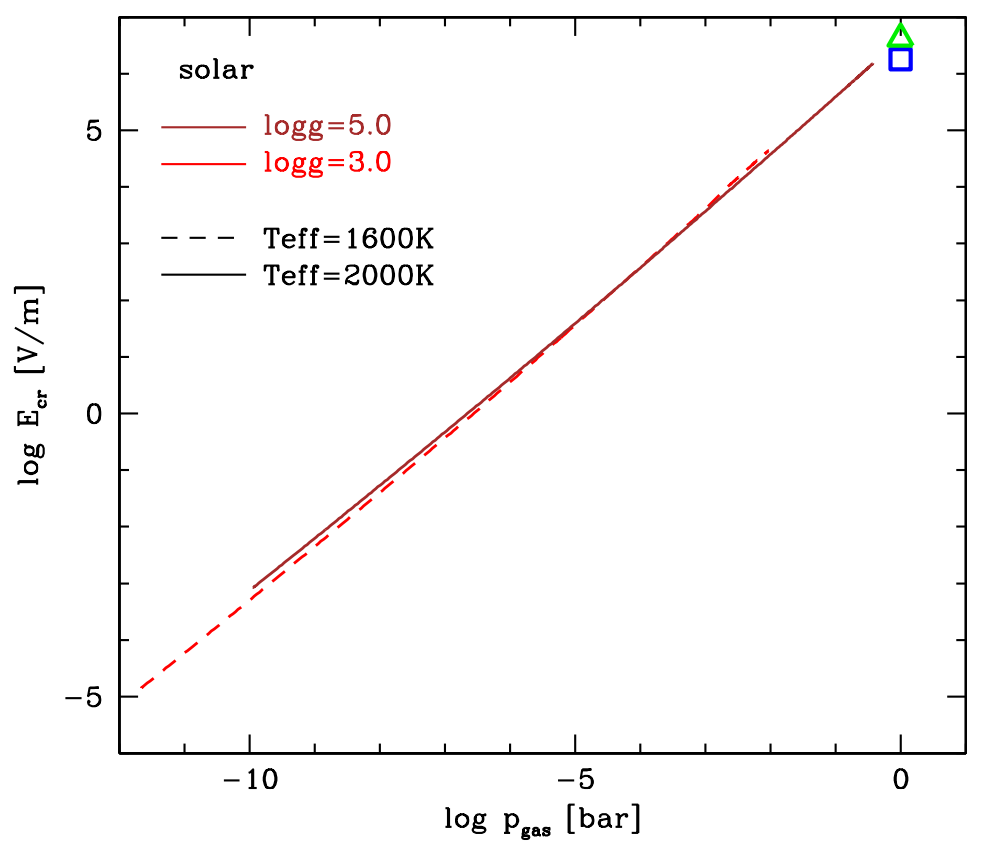

Figure 5: The break-down field strengths, $E_{\text {crit }}$, for three different brown dwarf and giant gas planet DrIFT-Phoenix model atmosphere of an initial solar element composition: $T_{\text {eff }}=1600 \mathrm{~K}, \log (\mathrm{g})=5.0$ (brown dashed line; brown dwarf), $T_{\text {eff }}=1600 \mathrm{~K}, \log (\mathrm{g})=3.0$ (red dashed line; giant gas planet), $T_{\text {eff }}=2000 \mathrm{~K}, \log (\mathrm{g})=5.0$ (brown solid line; brown dwarf). The break-down field is calculated for a $\mathrm{H}_{2}$-rich gas in the model atmosphere simulations of initial solar element abundances. Values for Earth (green triangle) and Jupiter (blue square) at 1 bar are shown for comparison.

Figure 5 shows the electric field that needs to be overcome by a mineral cloud to initiate a lightning-discharge. The values for Earth (green triangle) and for Jupiter (blue square) are shown for comparison and pressure unit check. Note that these values follow the classical break-down field parameterisation as given in Yair et al. (1995) but that field measurements above thunderclouds suggest an electric break-down field of 1-2 orders of magnitude less. Clearly, the break-down is much easier in low-pressure regimes which is apparent from Fig. 5 for the studied model atmospheres. Note, however, that the Paschen curves shown in Fig. 5 would turn into the Paschen minimum if continued to lower pressures before they become unphysically asymptotic at very low pressures.

For a given chemical composition, the break-down field is a strong function of gas pressure, therefore it is of similar orders of magnitude for all 
models which differ in gravity and effective temperature. All model simulations are performed for a hydrogen-dominated gas, with $\mathrm{H}_{2}$ being the main ionised species. Hydrogen remains the most abundant molecule also in the cloud forming part of the atmosphere where far less abundant elements (e.g. Si, Fe, Mg, O) are depleted by dust formation (Fig. 4 in Helling et al. 2008a).

We conclude that mineral clouds should also be able to produce discharge events as the break-down field are considerably lower than on Earth and Jupiter $\left(\sim 10^{6} \mathrm{~V} / \mathrm{m}\right)$ in the cloud forming regions. We suggest that lightning discharges should be expected in the upper part of the atmosphere and the mineral cloud, as gravitational settling provides a mechanisms for large-scale charge separation. Particles of different sizes fall with different velocities which leaves the smaller, less negatively charged (e.g. Merrison et al. 2012) cloud particles suspended for longer in the upper cloud layers. We reached a similar conclusion in a previous paper (Helling, Jardine \& Mokler 2011) where we compared streamer timescales and Coulomb recombination time scales with the time scale on which dust particles pass through a previously formed electron cloud (streamer) and, hence, potentially initiate further electron avalanches. Our understanding is that a superposition of avalanche-streamer processes will lead to more and more free electrons for a short time period which then may be defined as lightning. Such a superposition is more likely in the upper, low-pressure part of the cloud, too.

How much charges do we need to achieve an electric field break down? Treating a cloud as a capacitor allows to approximate the number of charges per $\mathrm{m}^{2}, Q_{\mathrm{cr}} / A=E_{\mathrm{cr}} \cdot \epsilon_{0}\left(\epsilon_{0}\right.$ - electric constant $)$, needed to overcome the break-down field. Figure 6 shows the number of charges per surface area needed to overcome the break-down field is strongly height-dependent in the atmosphere. The atmospher's height is represented by the pressure scale in Fig. 6: high pressures refer to lower atmospheric heights with high densities, and low pressures refer to the higher atmospheric layers with low densities. The number of charges per surface area in Fig. 6 is compared with the pressure regime in which dust-dust collisions are most likely producing charged dust particles. Our results suggest that dust plays an important role in producing free charges in most of the cloud of cool brown dwarfs, for example by triggering electron avalanches. Only a fraction of the cloud can be charged by dust-dust collisions in hotter brown dwarfs and in the less-dense giant gas planet atmospheres. Note, however, that the cloud in a giant gas planet is $100 \times$ more extended than the clouds forming in brown dwarf atmospheres 


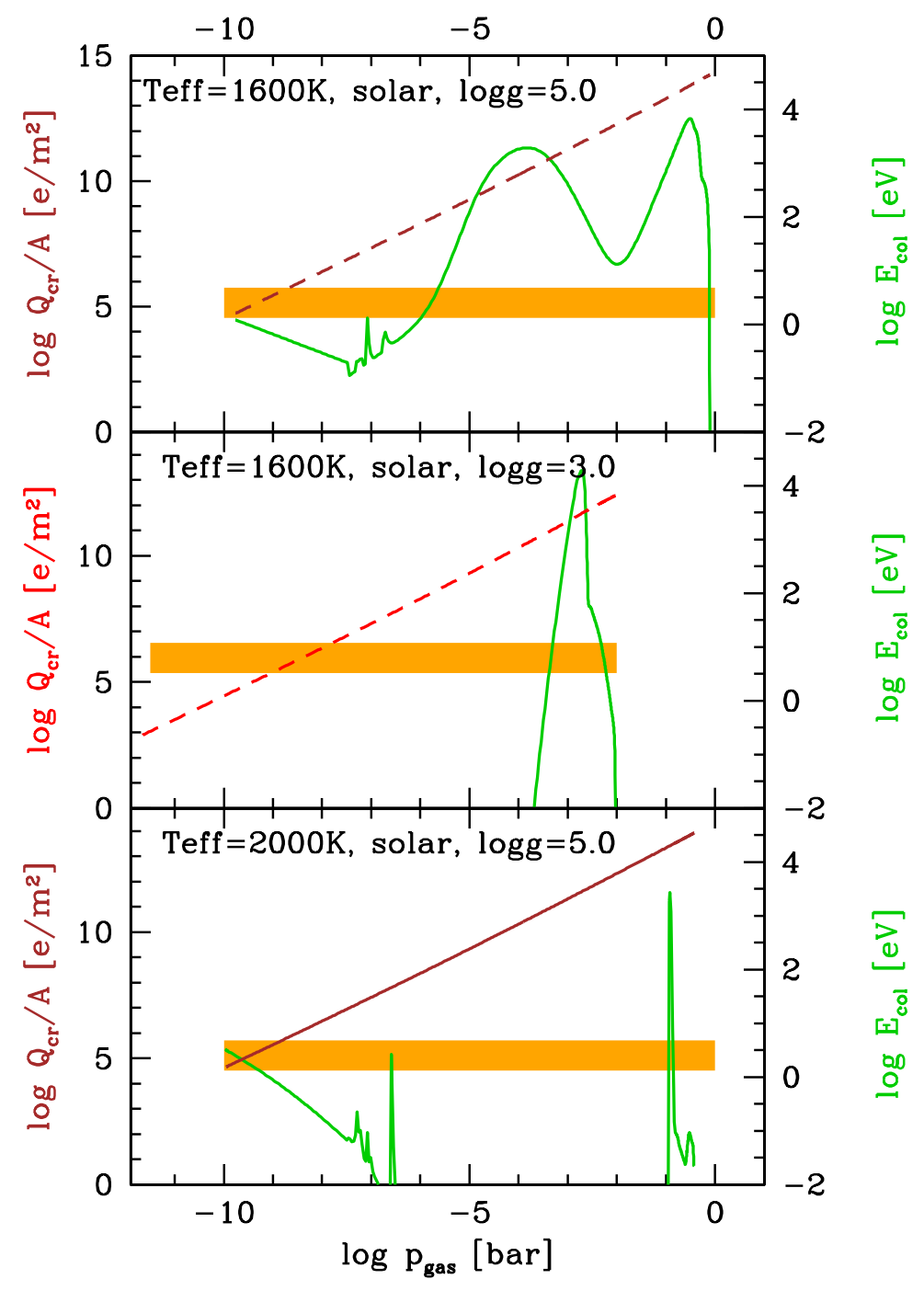

Figure 6: Number of charges per $\mathrm{m}^{2}$ (red and brown lines; left axis) needed to produce the break-down field shown in Fig. 5. The overplotted (green; right axis) lines is the energy produced during dust-dust collisions as in Fig. 2. The orange bar indicates the interval of work functions of different materials. This figure demonstrate where dust-grains can be charged by intra-cloud collisional processes compared to the distribution of needed breakdown charges inside the cloud. Note that the geometrical extension of the cloud differs between brown dwarfs and gas giants which is not reflected by the pressure scale shown in this plot. The comparison is shown for the same three atmosphere models like in Fig. 5. 
with high surface gravity.

\section{Conclusions}

Assuming that cloud particles are charged in brown dwarf and exoplanetary atmospheres, then electron avalanche processes are initiated between two charged grains and develop to a streamer's ionisation front (Helling et al. 2011a). We have argued that a large part of the clouds in brown dwarfs and extrasolar planets is susceptible to local discharge events which are triggered by charged dust grains. Such discharges occur on time scales shorter than the time required to neutralise the dust grains, and their superposition might produce enough free charges to suggest a partial and stochastic coupling of the atmosphere to a large-scale magnetic field. Discharge processes in brown dwarf and exoplanetary atmospheres should not connect to a crust as on terrestrial planets, hence, they will experience intra-cloud discharges comparable to volcano plumes and dust devils.

Acknowledgement: $\mathrm{ChH}$ acknowledges an ERC starting grant for the LEAP project of the EU program FP7 Ideas. Most of the literature search was done with the ADS. The computer support at our institutes is highly acknowledged.

\section{References}

[Berger et al. 2010] Berger E., Basri G., Fleming T.A., Giampapa M.S., Gizis J.E. et al. 2010, ApJ 709, 332

[Christensen et al. 2009] Christensen U.R., Holzwarth V., Reiners A. 2009, Nature, 457, 167

[Dehn 2007] Dehn M 2007, PhD Thesis, University Hamburg

[Dolginov et al 1993] Dolginov A.Z., Stepinski T.F. 1993, ApJ 427, 377

[Dowds et al. 2003] Dowds B.J., Barrett R.K., Diver D.A. 2003, Phys Rev E, $68(2), 6412$

[Glauser et al 2009] Glauser A.M., Güdel M., Watson D.M., Henning T., Schegerer A.A. et al. 2009, A\&A 508, 247 
[Hallinan et al. 2008] Hallinan G., Antonova A., Doyle J.G., Bourke S., Lane C., Golden A. 2008, ApJ 684, 644

[Helling et al. 2011a] Helling Ch., Jardine M., Witte S., Diver D. 2011, ApJ 727,46

[Helling et al. 2011b] Helling Ch., Jardine M., Mokler F. 2011, ApJ 737, 38

[Helling \& Rietmeijer 2009] Helling Ch. \& Rietmeijer F.J.M. 2009, IJAsB 8, 3

[Helling et al. 2008] Helling Ch., Ackerman A., Allard F., Dehn M., Hauschildt P.H. et al. 2008, MNRAS 391, 1854

[Helling et al. 2008a] Helling Ch., Dehn M., Woitke P., Hauschildt P.H. 2008, ApJ L675, 105

[Helling et al. 2008b] Helling Ch., Woitke P, Thi W.-F. 2008, A\&A 485, 547

[Helling \& Woitke 2006] Helling Ch. and Woitke P. 2006, A\&A 455, 325

[Helling et al. 2001] Helling Ch., Oevermann M., Lüttke M.J.H., Klein R., Sedlmayr E. 2001, A\&A 376, 194

[Jardine \& Cameron 2008] Jardine M., Cameron A.C. 2008, A\&A 490, 843

[Jones \& Tsuji 1998] Jones H.R.A. \& Tsuji T. 1997, ApJ 480, 39

[Koskinen et al. 2010] Koskinen T.T., Cho J.Y.-K., Achilleos N., Aylward A.D. 2010, ApJ 722, 178

[Merrison et al. 2012] Merrison J.P., Gunnlaugsson H.P., Hogg M.R., Jensen M., Lykke J.M., Madsen M.Bo, Nielsen M.B., Nørnberg P., Ottosen T.A., Pedersen R.T. et al. 2012 P\&SS 60, 328

[Mohanty et al 2002] Mohanty S., Basri G., Shu F., Allard F., Chabrier G. 2002, ApJ 571, 469

[Morin et al 2010] Morin J., Donati J.-F., Petit P., Delfosse X., Forveille T., Jardine M. M. 2010, MNRAS 407, 2269

[Nicoll \& Harrison 2010] Nicoll K.A. and Harrison R.G. 2010, GRL 37, L13802 
[Pont et al 2008 ] Pont F., Knutson H., Gilliland R.L., Moutou C., Charbonneau D. 2008, MNRAS 385, 109

[Patience et al. 2012] Patience J., King R.R., De Rosa R.J., Vigan A., Witte S., Rice W., Helling Ch., Hauschildt P.H. 2012, A\&A 540, 85

[Reiners \& Basri 2008] Reiners A., Basri G.2008, ApJ 684, 1390

[Saumond et al. 2006] Saumond D., Marley M.S., Cushing M.C., Leggett S.K., Roellig T.L. et al. 2006, ApJ 647, 552

[Sickafoose et al. 2001] Saunders C. 2008, SSRv 137, 335

[Sickafoose et al. 2001] Sickafoose A. A., Colwell J. E., Hornyi M., Robertson, S. 2001, JGR 106, 8343

[Sing et al. 2011 ] Sing D.K., Pont F., Airgrein S., Charbonneau D, Desert J.-M. et al. 2011, MNRAS 416, 1443

[Stephens et al. 2009] Stephens D.C., Leggett S.K., Cushing M.C., Marley M.S., Saumon D. et al. 2009, ApJ 702, 154

[Umebayashi \& Nakano 1981] Umebayashi T. and Nakano T. 1981, Publ. Astron. Soc. Japan 33, 617

[Witte et al. 2011] Witte S., Helling Ch., Barman T., Heidrich N., Hauschildt P.H. 2011 A\&A 529, 44

[Witte et al. 2009] Witte S., Helling Ch., Hauschildt P.H. 2009, A\&A 506, 1367

[Woitke\& Helling 2004] Woitke P. and Helling Ch. 2004, A\&A 414, 335

[Woitke \& Helling 2003] Woitke P. and Helling Ch. 2003, A\&A 399, 297

[Yair et al. 1995] Yair Y., Levin Z., Tzivion S. 1995, Icarus 115, 421

[Zarka et al. 2001] Zarka P., Treumann R.A., Ryabov B.P., Ryabov V.B. 2001, Ap\&SS 277, 293 